\title{
Análisis de la introducción del vehículo eléctrico en las flotas de transporte de carga leve: estudio de caso de la empresa brasilera Correios ${ }^{\circledR}$
}

\author{
Elisa A. Pereira \\ Empresa Brasilera de Correios e Telégrafos (CORREIOS $®)$ \\ Juan P. E. Gomez \\ Universidad de Brasília (UnB), Programa de Posgrado en Transportes-PPGT \\ Fabiana S. de Arruda \\ Universidad de Brasília (UnB), Programa de Posgrado en Transportes-PPGT
}

\section{RESUMEN}

Uno de los desafíos ambientales del mundo consiste el de reducir los problemas causados por las emisiones de las emisiones de Gases de Efecto Invernadero (GEI) provenientes principalmente de los Vehículos de Combustión Interna (VCI) que representan alrededor del $40 \%$ del crecimiento del dióxido de carbono $\left(\mathrm{CO}_{2}\right)$ en el mundo, lo hizo que varios países y por consiguiente algunas empresas, se movilizaran en torno a un compromiso a gran escala para respaldar las contribuciones empresariales a la promoción de una economía verde e inclusiva. El Vehículo Eléctrico (VE) surge como una de las alternativas más eficientes para reducir la contaminación causada por el transporte. Por lo anteriormente mencionado, este estudio tiene como objetivo analizar el VE utilizado para llevar a cabo servicios de entrega postal y distribución de objetos y encomiendas en Brasil por medio de la empresa SEDEX, para lo cual, se eligió un VE de propiedad de la empresa Correios que opera en el Distrito Federal en un recorrido de aproximadamente $110 \mathrm{~km}$, operado de lunes a viernes, con el fin de observar su idoneidad operativa, especialmente en lo referente a motor y sistema eléctrico, frenos, manipulación y ergonomía. Fueron evaluados el ruido del motor y de la suspensión en relación con la capacidad de absorción de las irregularidades del pavimento (impacto/vibración), así como la velocidad, la aceleración y rendimiento en las carreteras, la potencia del motor para mantener las pendientes medias de velocidad crecientes, así como su velocidad media. Finalmente fue evaluada la autonomía del VE con el fin de verificar su adhesión a la ejecución de las actividades postales. Los hallazgos más importantes de la investigación se atribuyen a la disminución de las emisiones de gases de efecto invernadero, ruido y vibraciones, además de una mayor eficiencia en las maniobras debido sobre todo al frenado progresivo.

\section{INTRODUCCIÓN}

En el siglo actual serán necesarias innovaciones tecnológicas, económicas, sociales e institucionales con el fin de crear perspectivas de reducción de emisiones contaminantes. Una de las principales innovaciones son las que dan como resultado la reducción de emisiones de $\mathrm{CO}_{2}$, concentrándose en la eficiencia energética en sectores clave como el transporte y la industria del (IPCC, 2014). En la industria automotriz europea y americana, los combustibles provienen generalmente de energía limpia como biodiesel, gas natural, etanol y energía eléctrica, este último, aún sin ser considerado como combustible, ha sido ampliamente utilizado en este sector (Grant-Muller y Usher, 2014; Dijk, et al., 2013; Lima y De Sousa, 2015). Este escenario ha dado lugar a una serie de acuerdos multilaterales con 
el fin de desarrollar políticas de conservación y desarrollo sostenible en el mundo (Wan et al., 2015).

Algunos acuerdos de importancia han sido firmados desde principios de los años 70, algunos de los cuales se destacan la Conferencia de las Naciones Unidas sobre el Medio Ambiente Humano (Declaración de Estocolmo, 1972), la Comisión Mundial sobre el Medio Ambiente y el Desarrollo (Informe Brundtland, 1987), la Declaración de Río sobre el Medio Ambiente y Desarrollo, Río 92 (Declaración, 1992). Sin embargo, la convención más referente a la reducción de las emisiones de $\mathrm{CO}_{2}$ fue la de Kyoto, Japón, firmada en diciembre de 1997 de donde surgió, la Conferencia de las Partes (COP-3) donde se firmó el compromiso de cada uno de los países adheridos al acuerdo para reducir sus emisiones combinadas de GEI en al menos un 5\% respecto a los niveles de 1990 durante el periodo 2008-2012 (Protocolo, 1998).

En la Conferencia de las Naciones Unidas sobre el Desarrollo Sostenible Río+20 fue firmado el compromiso "El futuro que queremos", por los países involucrados en el apoyo al uso de diversas fuentes de energía que aseguren así la protección del medio ambiente, especialmente con el uso de energías limpias y otras fuentes de baja emisión de GEI (ONU, 2012; Informe de Río+20, 2012). En Brasil, El Pacto Global Red Brasilera, fruto del compromiso firmado, ha servido como un compromiso a gran escala con la participación de más de 200 consejos de representantes de las empresas brasileras que han respaldado la promoción de una economía verde e inclusiva, desarrollando el establecimiento de compromisos específicos sostenibles (Rio+20, 2012).

Para Samaras y Meisterling (2008), uno de los desafíos para reducir los problemas ambientales es la reducción de emisión de GEI, principalmente provenientes de VCI, los cuales representan alrededor del $40 \%$ del crecimiento de dióxido de carbono $\left(\mathrm{CO}_{2}\right)$ en el mundo desde 1990. Los compromisos anteriores tienen como piedra angular la preocupación por los impactos medioambientales de las actividades que dan lugar a una cantidad significativa de las emisiones de GEI, como causantes de enfermedades respiratorias, enfermedades del corazón, además de agravar las ya existentes y aumentar las tasas de cáncer, factores estos que generan impactos negativos sobre la salud (Becker et al., 2009).

En este escenario, para Rocha (2013), el VE aparece como una tecnología alternativa innovadora, que se presenta como un medio de transporte ambientalmente eficiente, debido principalmente al uso de energías limpias. En Brasil, según Da Cunha (2011), el 87\% de la energía limpia proviene de centrales hidroeléctricas $(80 \%)$, bagazo de caña $(4 \%)$, nuclear $(2 \%)$ y el viento ( $1 \%)$. Por el contrario, el VCI utiliza como fuente de energía, combustibles fósiles que son contaminantes y contribuyen a la emisión de GEI (Mazon, et al., 2013).

Este artículo tiene como objetivo evaluar la eficiencia de VE de transporte de carga leve (800 kg) utilizados en la distribución de correo dentro de la empresa Brasilera de Correos y Telégrafos "Correios ${ }^{\circledR}$ ", los impactos ambientales y financieros del uso este tipo de tecnología en relación con el VCI, utilizando como método un estudio de caso de la flota de VE de la empresa Correios ${ }^{\circledR}$. Por lo tanto, en un principio será presentada una contextualización teórica del concepto de vehículos eléctricos en el mundo y en Brasil, seguido de estudio de caso, el análisis de los resultados y por último, las consideraciones finales.

\section{REFERENCIAL TEÓRICO}




\subsection{Energía y vehículos eléctricos}

En Brasil, el sector del transporte consume el 33,9\% de la energía producida, y el 32\% de los combustibles fósiles (BEN, 2014), es decir, después del sector industrial, el sector del transporte es la segunda mayor fuente de consumo de energía (Rocha, 2013; Baran y Loureiro, 2010), y la mayor fuente contaminante móvil de Brasil (Mazon et al, 2013), convirtiéndose en la principal causa de enfermedades cardiorrespiratorias.

Ya en el escenario mundial, es evidente la dependencia energética del petróleo, lo que genera un agotamiento de las de fuentes de energía primaria y graves impactos ambientales (Ribeiro et al., 2012; Rocha, 2013; Baran y Loureiro, 2010). Este proceso provoca la construcción de nuevos modelos de uso de energía sustentable para crear políticas de movilidad, cuyo principal objetivo es reducir el uso de recursos no renovables y la mejora de la calidad de vida de la población (Mazon et. al., 2013).

En esta perspectiva, el VE surge como una alternativa, con importantes beneficios ambientales, debido principalmente al uso de energías limpias (Rocha, 2013), diferentes al VCI que utiliza combustibles fósiles, que aumentan las emisiones de contaminantes que contribuyen a la emisión de GEI (Mazon, et al., 2013).

Para Almeida et al., (2015) y Fender (2011), los VE, además de contar con un rendimiento medioambiental más favorable, cumplen con los principios de la ergonomía, debido principalmente al hecho de que producen menos vibraciones y son más silenciosos, lo que proporciona una mejor calidad de vida para los conductores de este tipo de vehículos.

\subsection{Ergonomía y vehículos eléctricos}

Estudios muestran que la industria del transporte por carretera representan un alto riesgo para la salud física y mental de los conductores (Penteado et al., 2008; Battiston y Hoffmann, 2006; Neri et al., 2005), lo que ha sido presentado por la Organización Mundial de la Salud en su informe sobre la situación mundial de la seguridad del tráfico en 2013 (OMS, 2013) en donde se demostró que el número de accidentes de tráfico en Brasil saltó de 19 por cada 100.000 habitantes a 23,4 por cada 100.000 habitantes, convirtiéndose en el registro más alto en América del Sur.

El su entorno de trabajo, el conductor sufre presiones internas y externas, siendo estos factores de riesgo que pueden precipitar la ocurrencia de un eminente accidente de tráfico. En este sentido, Hoffmann (2000) afirma que entre las presiones externas se destacan las exigencias del tránsito (ambiente) y entre las presiones internas, las condiciones ergonómicas del vehículo como la posición del motor, precariedad mecánica, además de ruido y vibraciones.

Los motores de los VE según Rocha (2013), son más pequeños que el motor de un VCI y completamente en silencios, sin embargo, poseen una velocidad máxima reducida, como es relatado en la ficha técnica del vehículo de marca Renault Kangoo, $800 \mathrm{~kg}$, donde la velocidad máxima informada corresponde a $59 \mathrm{~km} / \mathrm{h}$ utilizando gasolina, $161 \mathrm{~km} / \mathrm{h}$ con etanol y $130 \mathrm{~km} / \mathrm{h}$ electricidad, además Santamarta (2009) afirma que el motor del VE es considerado cuatro veces más eficiente que el motor de los VCI.

Dentro de la infraestructura de transporte, según fue relatado por Almeida et al., (2015), los vehículos de transporte de carga leve utilizados para llevar a cabo servicios de recogida y entrega de correo representan una oportunidad con respecto a la reducción de emisiones, 
debido a que estas operaciones podrían llevarse a cabo con vehículos eléctricos como el testado por la empresa Correios ${ }^{\circledR}$. La flota de los vehículos de carga leve dentro de la empresa Correios ${ }^{\circledR}$ está compuesta por 3.724 VCI (Almeida et al., 2015). Dentro de esta perspectiva, la empresa $\operatorname{Correios}^{\circledR}$, así como otras empresas de servicios postales y de entrega urgente del mundo empezaron a introducir el VE en sus flotas.

Finalmente, y no menos importante que las bajas emisiones de GEI, los VE suelen mostrar menos vibraciones, ruido por la suspensión en relación a la capacidad de absorción de las irregularidades del pavimento (impacto/vibración), eventualidades que son transmitidas directamente al conductor (Almeida et al., 2015; Fender, 2011).

\subsection{Inserción del vehículo eléctrico en actividades postales}

El transporte de carga, de acuerdo Crainic (2000) es un componente vital de la economía de un país, sobre todo porque sirve de apoyo a las actividades de producción, comercio y consumo, garantizando la circulación eficiente y disponibilidad de materias primas y productos terminados.

En el mundo hay una creciente competencia en el mercado postal y empresas de remesas expresas, lo que ha llevado a volver a evaluar y mejorar continuamente los procesos de negocio. En los mercados postales europeos, esta tendencia es aún más amplia gracias a los esfuerzos actuales de la desregulación y la privatización de la Unión Europea y los gobiernos en el medio y los países de Europa del Este (Grünert y Sebastian, 2000).

En Brasil los envíos postales son atendidos en un $100 \%$ por la Empresa Correios ${ }^{\circledR}$ en relación a los servicios de cartas, telegramas y paquetes de pequeño porte. Ya en los servicios de envíos expresos donde se da una amplia competencia, se observó que la cuota de mercado (penetración del servicio de mercado) para el mes de diciembre de 2015, de acuerdo con el informe de ejecución del servicio, la empresa Correios ${ }^{\circledR}$ cumplió con aproximadamente el $80 \%$ de los envios expresos.

De acuerdo con Almeida et al. (2015), dentro de los objetivos ambientales, la empresa Correios ${ }^{\circledR}$ en la Conferencia Río +20 se comprometió en reducir el $12 \%$ de sus emisiones de $\mathrm{CO}_{2}$ durante la ejecución de sus actividades postales hasta el año 2020. Una de las formas utilizadas para lograr este objetivo, fue precisamente la implantación del vehículo eléctrico en sus flotas de distribución.

Almeida et al., (2015) encontraron en su estudio que Brasil tiene un escenario favorable para la inserción de la VE en la sociedad, siendo los órganos públicos, en los que se incluyen la empresa $\operatorname{Correios}{ }^{\circledR}$, los principales instigadores de la incorporación de esta tecnología en su flotas, ya que el país cuenta con una amplia red de distribución de energía eléctrica y un alto potencial de generación a partir de fuentes limpias en constante renovación, tales como la construcción de nuevas centrales hidroeléctricas en el país.

\section{ESTUDIO DE CASO}

Dentro de la estructura de los transportes, la empresa Correios ${ }^{\circledR}$ cuenta actualmente con una flota de 23.400 VCI utiliza para realizar las actividades postales, de los cuales 3.724 son vehículos de carga ligeros de marca Renault Kangoo con capacidad de carga de $800 \mathrm{~kg}$, utilizados en este estudio para estimar el inventario de $\mathrm{CO}_{2}$. Las mismas variables utilizadas para medir las emisiones de VCI se aplicaron a los VE, ya que los dos vehículos tienen las 
mismas características en relación con la marca y el modelo, lo que garantizó un análisis comparativo libre de posibles errores.

En un estudio realizado por Almeida et al., (2015) en la empresa Correios ${ }^{\circledR}$, se adoptaron análisis comparativos de la evolución de los impactos medioambientales de los VCI y los $\mathrm{EV}$, en donde se midieron emisiones de $\mathrm{CO}_{2}$, mantenimiento y abastecimiento. Entre las principales conclusiones se observó una variación de más de siete veces el costo del consumo por cada $100 \mathrm{~km}$. La relación de emisión de $\mathrm{CO}_{2}$ comparado entre un VE y un VCI mostró una diferencia significativa, ya que las emisiones de $\mathrm{CO}_{2}$ de los VCI resultaron 163 veces más alta que las de un VE.

En el mismo estudio, fue demostrado que la autonomía de estos vehículos ya no es un impedimento en el servicio de entrega postal en el país y se convierte en una variable a considerar en la planificación con el fin de crear rutas adecuadas a sus características.

\section{ANALISIS DE LOS RESULTADOS}

Teniendo en cuenta que los vehículos analizados se componen por un VCI bi-combustíble (Flex) y un VE (en etapa de prueba), resulta importante aclarar que la empresa Correios ${ }^{\circledR}$ elige el tipo de combustible usado en los vehículos Flex (Gasolina o Alcohol), que le ofrezca las mejores condiciones financieras a la empresa, de acuerdo con la relación de consumo. La base de datos utilizada para calcular el consumo fue referente a los meses de abril y mayo para el VE y junio y julio para el vehículo Flex (los cuatro meses corresponden al año 2015). En este sentido, el combustible para el vehículo Flex que la empresa Correios ${ }^{\circledR}$ consideró más ventajoso financieramente durante estos meses fue gasolina.

Con el fin de estudiar la adhesión a la logística de distribución de la empresa $\operatorname{Correios}^{\circledR}$ fue testado un VE en la ruta entre el sur y el norte de Brasilia (Plano Piloto) lo que equivale a un promedio de 92,91 kilómetros/día rodados conforme a lo presentado en la Figura 4.1 con una frecuencia lunes y viernes. La ruta recorrida por el VE es la ruta más larga en kilómetros de distribución de los envíos postales del Distrito Federal. Esta ruta fue creada incialmente para ser realizada por un VCI.

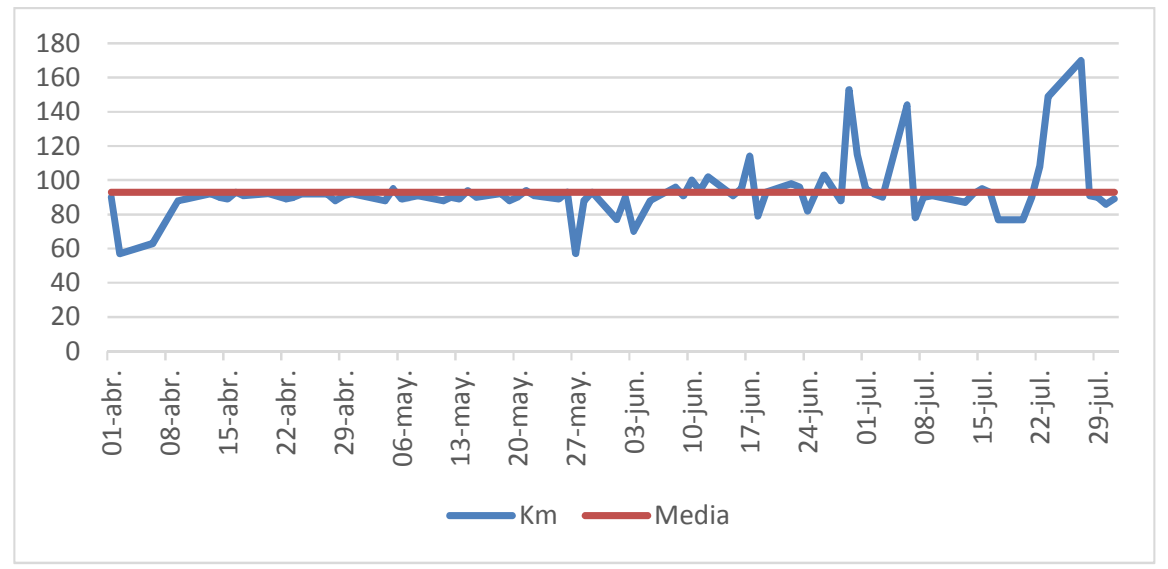

Fig. 4.1 Kilómetros rodados por día

Se puede observar en la Figura 4.1 el desempeño de ambos vehículos evaluados (VE y VCI), en donde la autonomía del VE cumplido con el itinerario establecido por la empresa Correios ${ }^{\circledR}$ en la ruta estudiada durante el tiempo analizado. 
Da Cunha (2011), afirma que la baja autonomía de los VE no les permite viajar más de 200 $\mathrm{km}$, incluso con sus baterías completamente cargadas, lo cual es resulta ser una característica que preocupa a los futuros compradores. También, de acuerdo con el autor, el tener que parar para recargar las baterías durante largos periodos de tiempo (de 4 a 8 horas), convierte a los VE en una opción poco viable.

La autonomía de los vehículos, medida en este estudio por el consumo de combustible promedio de $92.91 \mathrm{~km} /$ día andados por cada uno de los vehículos analizados (Tabla 4.1), se basa en el costo de compra de $\$ 3,53$ reales por litro de gasolina y de $\$ 0,55$ reales por $\mathrm{kW}$ de electricidad (datos suministrados por la empresa $\operatorname{Correios}^{\circledR}$ ).

\begin{tabular}{cccc} 
Tabla 4.1 Costos de abastecimiento de la empresa & Correios ${ }^{\circledR}$ (média/día) \\
\cline { 2 - 4 } Vehículo & Consumo para & Costo & Costo Total \\
& $92,91 \mathrm{~km} /$ dia & $(\mathrm{R} \$)$ & $(\mathrm{R} \$ / 92,91 \mathrm{~km})$ \\
\hline VCI & $15,78 \mathrm{~L}$ & $\mathrm{R} \$ 3,53 \mathrm{~L}$ & $\mathrm{R} \$ 55,70$ \\
$\mathrm{VE}$ & $14,29 \mathrm{~kW}$ & $\mathrm{R} \$ 0,55 \mathrm{~kW}$ & $\mathrm{R} \$ 7,86$ \\
\hline
\end{tabular}

La Tabla 4.2 muestra los resultados obtenidos por el análisis comparativo de los datos para el período de referencia (abril a julio de 2015) entre un VE y un VCI, manteniendo las mismas especificaciones técnicas (marca, modelo y referencia) y las mismas cualidades funcionales (itinerario). Se mantuvo siempre la misma variable de consumo de los dos vehículos para el análisis (7526 kilómetros por período). Se puede observar que el comportamiento de los gastos de abastecimiento del VCI es $708 \%$ más caro que el VE.

En la misma tabla, fue utilizado basado en el factor de conversión de GEI proporcionada por el Ministerio de Ciencia y Tecnología de Brasil. Por lo tanto, se aplica a su factor de conversión de referencia (2.212 kg para gasolina y de 0,1355 para energía eléctrica) para el periodo de estudio. Como resultado, se llegó a las toneladas de $\mathrm{CO}_{2}$ emitido por el vehículo de transporte de carga leve. Se puede observar que el comportamiento de las emisiones de $\mathrm{CO}_{2}$ de los VCI son $1.802 \%$ más altas que las de un VE.

Tabla 4.2: Costos de de abastecimento de la empresa Correios ${ }^{\circledR}$ (média/período)

\begin{tabular}{ccccc}
\hline Vehículo & $\begin{array}{c}\text { Consumo para } \\
7526 \mathrm{~km}\end{array}$ & $\begin{array}{c}\text { Costo } \\
(\mathrm{R} \$)\end{array}$ & $\begin{array}{c}\text { Costo Total } \\
(\mathrm{R} \$ / 7526 \mathrm{~km})\end{array}$ & $\begin{array}{c}\text { Total } \mathrm{CO}_{2} \\
\text { emisiones }(\mathrm{kg})\end{array}$ \\
\hline $\mathrm{VCI}$ & $1.278,22 \mathrm{~L}$ & $\mathrm{R} \$ 3,53 \mathrm{~L}$ & $\mathrm{R} \$ 4.512,11$ & $2.827,42$ \\
$\mathrm{VE}$ & $1.157,53 \mathrm{~kW}$ & $\mathrm{R} \$ 0,55 \mathrm{~kW}$ & $\mathrm{R} \$ 636,64$ & 156,84 \\
\hline
\end{tabular}

En el presente estudio se aplicó también un cuestionario a los conductores de VE de la empresa Correios con el fin de evaluar el desempeño del vehículo en prueba en la actividad postal. El cuestionario se realizó, basado en la perspectiva del usuario en relación con: motor, sistema eléctrico, la eficiencia de frenado, maniobrabilidad y ergonomía. La muestra se compone de cinco encuestados que corresponden a los conductores capacitados para conducir el vehículo de prueba. Este hecho hace que la muestra sea reducida para realizar un análisis estadístico.

Aun con una muestra reducida, los cuestionarios muestran la percepción del usuario (conductor) en aspectos importantes del uso de un VE, tales como la reducción del ruido del motor y la suspensión en relación con la capacidad de absorción de impactos y las vibraciones resultantes de las irregularidades del pavimento, así como la velocidad, la 
aceleración y rendimiento de los caminos, la potencia del motor para las subidas como la velocidad media.

\section{CONSIDERACIONES FINALES}

Brasil tiene un escenario favorable para la inserción de VE en la sociedad, ya que cuenta con una amplia red de distribución de energía eléctrica y un alto potencial de generación a partir de fuentes limpias en constante renovación, como es el caso la construcción de nuevas centrales hidroeléctricas en el país. La electricidad generada en estas condiciones presenta ventajas medioambientales, ya que emite bajos niveles de $\mathrm{CO}_{2}$, en comparación con los emitidos por los combustibles fósiles.

Los beneficios ambientales hacen de los VE una solución atractiva en busca no sólo de disminuir los GEI, sino también sus implicaciones negativas para el medio ambiente. Además, estos vehículos tienen un bajo costo de mantenimiento y suministro en comparación con los vehículos convencionales. La autonomía de estos vehículos ya no es un impedimento, y se convierte en una variable a considerar en la planificación con el fin de crear rutas adecuadas a sus características.

Como se evidencia en este trabajo, el VE introducido en la mayor ruta de distribución de objetos postales de Brasilia, correspondiente a $92.91 \mathrm{~km} /$ día en media, viajado todos los días hábiles de la semana (lunes a viernes). Se encontró que el rendimiento del VE en la ruta establecida resulta adherente, toda vez que cumplió con las mismas rutas de un VCI, sin presentar ninguna falla mecánica.

El VE también resultó económicamente más ventajoso en comparación con el VCI, ya que el comportamiento de los costos de suministro de la VCI es $708 \%$ más caro que VE, además, con respecto a la contaminación producida, se demostró que el comportamiento del VCI respecto a emisiones de $\mathrm{CO}_{2}$ es $1.802 \%$ mayor que la del vehículo eléctrico.

Contrario a las afirmaciones Da Cunha (2011) en referencia a la baja autonomía del VE, en la operación de distribución de correos, este tipo de tecnología, presenta un comportamiento adherente a las actividades postales y abre una suposición de que el VE sea probado en rutas mayores, independientemente de las limitaciones de su autonomía, siendo capaz de planificar la logística, respetando las características de este tipo de vehículo.

Para futuros trabajos, varios estudios con este tema deben ser desarrollados en las empresas que utilizan VE en sus flotas (vehículos ligeros) con el fin de verificar que la percepción de los beneficios de este tipo de tecnología es similar a la encontrada en este estudio, así como es recomendado extender la muestra en relación con la inserción del VE a través de otro tipo de instrumento para la recolección de datos utilizado en muestras pequeñas tales como entrevistas, con el fin de medir y confirmar la evidencia de esta investigación para el grado de satisfacción del usuario en relación con la ergonomía del VE.

\section{Agradecimentos}

Los autores agradecen a la empresa de Correos y Telégrafos de Brasil Correios, por ceder los vehículos y la información para el trabajo desarrollado. 
CIT2016 - XII Congreso de Ingeniería del Transporte València, Universitat Politècnica de València, 2016. DOI: http://dx.doi.org/10.4995/CIT2016.2016.3383

\section{REFERENCIAS}

ALMEIDA, E. P., GOMEZ, J. P. E., DA SILVA SOUZA, A. C., \& DE ARRUDA, F. S. Análise comparativa dos custos dos veiculos de combustão interna e veículos elétricos: estudo de caso dos Correios.

BARAN, R.; LOUREIRO, L. F. (2010) "Veículos elétricos: história e perspectivas no Brasil." XIII Congresso Brasileiro de Energia, Brasil.

BEN. Balanço Energético Nacional. Relatório Síntese. Ministerio de Minas e Energía. 2014

BECKER, T. A., IKHLAQ S.; BURGHARDT T. (2009) "Electric vehicles in the United States: A new model with forecasts to 2030." Center for Entrepreneurship and Technology, University of California, Berkeley.

CRAINIC, T. G. (2000). Service network design in freight transportation.European Journal of Operational Research, 122(2), 272-288.

DA CUNHA. R. D., (2011) Análise da integração de veículos elétricos na matriz energética Brasileira. UFPA. Monografia.

DECLARAÇÃO, D. E. S. O. A. (1972). Conferência das Nações Unidas sobre Meio ambiente. Estocolmo.

DECLARAÇÃO, D. R. S. O. A. (1992). Conferência das Nações Unidas sobre Meio ambiente, Rio 92 . Rio de Janeiro.

DIJK, M., ORSATO, R. J., \& KEMP, R. (2013). The emergence of an electric mobility trajectory. Energy Policy, 52, 135-145.

FENDER, DAVID L. 2011. "Electric Vehicle Noise." Professional safety.

GRANT-MULlER, S., \& USHER, M. (2014). Intelligent Transport Systems: The propensity for environmental and economic benefits. Technological Forecasting and Social Change, 82, 149-166.

GRÜNERT, T., \& SEBASTIAN, H. J. (2000). Planning models for long-haul operations of postal and express shipment companies. European Journal of Operational Research, 122(2), 289-309.

HOFFMANN, M. H. (2000). Áreas de intervenção da Psicologia do Trânsito. Revista

IPCC (2014). Intergovernmental Panel on Climate Change Synthesis Report. Contribution of Working Groups I, II and III to the Fifth Assessment Report of the Intergovernmental Panel on Climate Change. Geneva

LIMA, N. C., \& DE SOUZA, G. H. S. (2015). A demanda do etanol e sua caracterização no mercado brasileiro de combustíveis. Organizações Rurais \& Agroindustriais, 16(4).

MAZON, M. T., CONSONI, F. L., QUINTÃO, R. (2013). Perspectivas para a implantação do veículo elétrico no Brasil: uma análise a partir do Sistema Nacional de Inovação e das redes colaborativas de C\&T, Congresso da Associação Latino-Americana de Gestão de Tecnologia. Vol. 1, pp.4140-4155, Porto, Portugal, 2013

NERI, M., SOARES, W. L., \& SOARES, C. (2005). Condições de saúde no setor de transporte rodoviário de cargas e de passageiros: um estudo baseado na Pesquisa Nacional por Amostra de Domicílios. Cad Saúde Pública, 21(4), 1107-23.

OMS Organização Mundial da Saúde Global Status Report on Road Safety 2013

ONU, (2012). The future we want Rio+ 20. Organização das Nações Unidas. Rio de Janeiro

PENTEADO, R. Z., DE OLIVEIRA GONÇALVES, C. G., DA COSTA, D. D., \& MARQUES, J. M. (2008). Trabalho e saúde em motoristas de caminhão no interior de São Paulo. Saúde e Sociedade, 17(4), 35-45.

PROTOCOLO. Q. S. M. C. (1998). Protocolo de Quioto à Convenção-Quadro das Nações Unidas sobre Mudança do Clima. Quioto.

RELATÓRIO BRUNDLAND (1987). Comissão mundial sobre meio ambiente e desenvolvimento. $2^{\mathrm{a}}$ Edição. Rio de Janeiro: Editora da FGV.

RIBEIRO, P., MENDES, J. F., \& RAMOS, R. A. (2012). Electric mobility in Portugal: municipal plans for its promotion. In EED'12-The 10th WSEAS International Conference on Environment, Ecosystems and Development (pp. 229-235). WSEAS Press.

RIO+20 Corporate Sustainability Forum. Innovation \& Collaboration Public Policy Recommendations Commitments to Action. Rio de Janeiro. 2012.

ROCHA, L. H. (2013). Carro Elétrico-Desafios para sua Inserção no Mercado Brasileiro de Automóveis. Tesis de doctorado. Universidad de São Paulo.

SAMARAS, C., \& MEISTERLING, K. (2008). Life cycle assessment of greenhouse gas emissions from plugin hybrid vehicles: implications for policy.Environmental science \& technology, 42(9), 3170-3176.

SANTAMARTA, J., (2009) "El futuro del automóvil es eléctrico." Técnica Industrial vol. 281, p. 26.

WAN, Z., SPERLING, D., \& WANG, Y. (2015). China's electric car frustrations. Transportation Research Part D: Transport and Environment, 34, 116-121. 